\title{
Restless legs syndrome and low brain iron levels in patients with haemochromatosis
}

\author{
J Haba-Rubio, L Staner, C Petiau, G Erb, T Schunck, J P Macher
}

J Neurol Neurosurg Psychiatry 2005;76:1009-1010. doi: 10.1136/jnnp.2003.030536

Regional brain iron levels of two patients with haemochromatosis and severe restless legs syndrome (RLS) were assessed using R2' magnetic resonance imaging (MRI) sequences in both patients and in nine healthy controls. R2' relaxation rates in the patients were decreased in the substantia nigra, red nucleus, and pallidum when compared with the controls. These results indicate that local brain iron deficiency may occur in patients with haemochromatosis and suggest a role for brain iron metabolism in the pathophysiology of RLS.

R estless legs syndrome (RLS) is characterised by an irresistible urge to move the legs, associated with unpleasant paraesthesias in the legs and sometimes in the arms. These sensations occur at rest, in particular in the evening or at night, and are relieved by movement. Many patients also have periodic limb movements in sleep (PLMS), and they may complain of insomnia and/or hypersomnia. ${ }^{1}$

Hereditary haemochromatosis is an autosomal recessive disease of iron metabolism in which increased intestinal absorption of iron leads to iron deposition in multiple organs. Neurological manifestations have been described rarely in hereditary haemochromatosis, including increased prevalence of headache, ${ }^{2}$ movement disorders, ${ }^{3}{ }^{4}$ and secondary dementia. ${ }^{5}$ Little is known about brain iron levels in these patients, although studies have suggested that some patients with haemochromatosis have increased iron deposition in the basal ganglia. ${ }^{6}$ Similarly, there are apparently no reports of an increased prevalence of sleep disorders, including RLS-PLMS.

The pathogenesis of RLS and PLMS remains largely unknown. Radiological and pharmacological findings have provided indirect evidence of dopaminergic system abnormalities. ${ }^{1}$ It has been suggested that this dysfunction can be mediated by low brain iron levels since iron is needed as a cofactor for tyrosine hydroxylase (the rate limiting enzyme in the synthesis of dopamine) and because the $\mathrm{D}_{2}$ receptor is a protein containing iron. ${ }^{7}$ Additional evidence comes from a magnetic resonance imaging (MRI) study that found decreased iron levels in the substantia nigra and, to a lesser degree, in the putamen of patients with idiopathic RLS. ${ }^{8}$ Cerebrospinal fluid (CSF) levels of ferritin are lower and transferrin levels higher in patients with RLS compared with age matched controls, despite normal serum levels of ferritin and transferrin. ${ }^{9}$ Furthermore, a neuropathological study has shown a defect in the regulation of the transferrin receptors in RLS, resulting in impaired iron acquisition by the brain. ${ }^{10}$

Here we report two patients with haemochromatosis with severe RLS and low brain iron levels, as measured by MRI.

\section{CASE REPORTS}

\section{Patient 1}

A 40 year old woman had experienced insomnia since early adulthood. She had no significant medical or surgical history.
At the origin of her insomnia was a compelling urge to move the limbs, usually associated with leg discomfort that the patient described "like electric current". These symptoms were exclusively present at rest, beginning after a delay of several minutes after the patient lay down and interfering with sleep onset. She had tried several hypnotics without lasting success.

Polysomnography demonstrated the presence of PLMS (index $=21.5$ movements/hour of sleep), mostly associated with microarousals. Routine laboratory analysis to rule out secondary RLS showed increased level of serum ferritin (305 ng/dl) and serum transferrin saturation (88\%). Detailed biochemical investigations were done with the genotyping of the HFE gene, which showed the C282Y mutation.

Clinically, the patient had no other organ manifestations. She was treated by venesection (initially twice a month, then once every three months), but there was no improvement in the sleep complaints. Clonazepam was then introduced, which diminished the RLS related symptoms and the associated insomnia.

She was not aware of RLS or any other case of haemochromatosis in her family. Her only brother was subsequently screened for haemochromatosis, and he was also homozygous for C282Y.

\section{Patient 2}

A 56 year old man was diagnosed as having haemochromatosis at the age of 53 years. Non-specific presenting symptoms (such as weakness, fatigue, apathy, and lack of libido) had appeared progressively approximately 18 months before the diagnosis. At that time, there were no significant clinical findings on the physical examination and the patient had no noteworthy medical or surgical history except for an episode of renal calculus 20 years previously. The patient was finally referred to the department of internal medicine of an academic hospital on the basis of an unexplained high level of blood ferritin, incidentally found on an extensive blood analysis. He was diagnosed as having hereditary haemochromatosis by a liver biopsy showing iron overload and a homozygous C282Y mutation on genotyping. The patient was not able to identify retrospectively any other case of haemochromatosis in his family.

Three years later, the patient was referred to our sleep disorder centre for a complaint of insomnia, non-restorative sleep, and daytime fatigue despite a regular phlebotomy regimen (that is, removal of $350 \mathrm{ml}$ of whole blood every two weeks). Typical symptoms of RLS with paraesthesia and dysesthesia in the legs relieved by movement were evidenced. The phlebotomies seemed to increase temporarily the symptoms of RLS and aggravate fatigue. Polysomnographic recordings, as well as MRI, were carried out three days after the phlebotomy, when the ferritin level was $46.8 \mathrm{ng} / \mathrm{dl}$, and showed an index of 123 PLMS/hour of sleep.

Abbreviations: PLMS, periodic limb movements in sleep; RLS, restless legs syndrome 


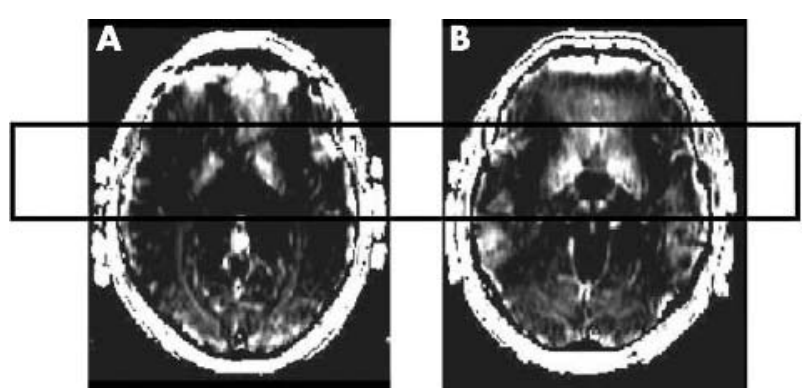

Figure 1 Quantitative analysis of different regions of interest obtained by calculating the relaxation rate R2' $\left(1 / T 2^{\prime}\right)$, a pure measure of focal field inhomogeneity due to tissue iron content. Basal ganglia R2' image in (A) a 56 year old patient with RLS and haemochromatosis (patient 2) and (B) a control subject.

Table 1 R2' values $(1 / \mathrm{msec})$ from brain regions of interest

\begin{tabular}{lllll}
\hline & \multicolumn{2}{l}{ Controls $(\mathbf{n}=9)$} & & \\
\cline { 2 - 3 } Region of interest & Median & Range & Patient 1 & Patient 2 \\
\hline Substantia nigra & 0.0105 & $0.0059-0.0198$ & 0.0037 & 0.0043 \\
Red nucleus & 0.0106 & $0.0059-0.0178$ & 0.0053 & 0.0040 \\
Putamen & 0.0094 & $0.0077-0.0149$ & 0.0102 & 0.0110 \\
Caudate & 0.0135 & $0.0066-0.0258$ & 0.0080 & 0.0284 \\
Pallidum & 0.0095 & $0.0077-0.0184$ & 0.0066 & 0.0052 \\
\hline & & & & \\
\hline
\end{tabular}

\section{Assessment of brain iron levels with MRI}

After obtaining written informed consent, we assessed the regional brain iron levels in both patients using a whole body 3 Tesla MRI system (Medspec S300, Brucker Medical, Germany). The MRI was done shortly after the polysomnographic recordings ( 15 days and two days, respectively). Effective transverse relaxation rates $\left(\mathrm{R} 2^{\prime}\right)$ were measured in different brain regions of interest (ROI) using a hybrid sequence combining one spin echo with 16 gradient echoes (fig 1). A set of 10 slices of $5 \mathrm{~mm}$ thickness and $1 \times 2$ resolution was acquired. R2' sequences have been shown to be an accurate, indirect, and quantitative non-invasive measure of brain iron levels. ${ }^{11}$ The results were compared with those from a group of nine healthy volunteers (five men, four women; mean (SD) age 48.1 (8.7) years, range 36-65) with no symptoms of RLS or primary sleep disorder, as assessed by polysomnography. R2' values were substantially decreased in the substantia nigra, red nucleus and pallidum in both patients compared with the controls (table 1), suggesting an iron deficit in these regions in the patients. These findings reveal that local brain iron deficiency may occur in patients with haemochromatosis, as if the bloodbrain barrier is able to restrict the transport of the excess plasma iron to the brain.
As regards RLS, we can only postulate that our patients represent sporadic associations between the two illnesses, in particular considering the elevated prevalence rates reported in the literature for RLS (ranging between 4\% and 15\%). ${ }^{1}$ But our MRI findings, similar to those reported by Allen et al in idiopathic RLS, ${ }^{8}$ indicate a possible role of local brain iron deficiency in the pathophysiology of RLS and PLMS, even in patients with systemic iron overload, such as in our patients. The discrepancy between serum and brain iron levels in patients with RLS further indicates that symptoms are not linked to low serum iron levels but to low regional brain iron availability. An alteration in the blood-brain barrier iron transport mechanism may be implicated in the pathophysiology of RLS symptoms. Further studies, analysing CSF ferritin and transferrin, and brain iron levels, are needed to confirm the hypothesis that an abnormal brain iron level is a primary mechanism of RLS.

\section{Authors' affiliations}

J Haba-Rubio, L Staner, G Erb, T Schunck, J P Macher, FORENAP

(Institute for Research in Neurosciences and Neuropsychiatry), Rouffach, France

C Petiau, Clinique Sainte Barbe, Strasbourg, France

Competing interests: none declared

Correspondence to: Dr J Haba-Rubio, Hôpitaux Universitaires Genève, Belle Idée, Chemin du Petit Bel-Air 2, 1225-Chêne-Bourg, Switzerland; Jose.HabaRubio@hcuge.ch

Received 16 October 2003

Revised version received 16 August 2004

Accepted 14 September 2004

\section{REFERENCES}

1 Hening W, Allen R, Earley C, et al. The treatment of restless legs syndrome and periodic limb movement disorder. An American Academy of Sleep Medicine Review. Sleep 1999;22:970-99.

2 Hagen K, Stovner $\amalg$, Asberg A, et al. High headache prevalence among women with hemochromatosis: the Nord-Trondelag health study. Ann Neurol 2002;51:786-9.

3 Demarquay G, Setiey A, Morel Y, et al. Clinical report of three patients with hereditary hemochromatosis and movement disorders. Mov Disord 2000; 15:1204-9.

4 Costello DJ, Walsh SL, Harrington HJ, et al. Concurrent hereditary haemochromatosis and idiopathic Parkinson's disease: a case report series. J Neurol Neurosurg Psychiatry 2004;75:631-3.

5 Harvey RJ, Summerfield JA, Fox NC, et al. Dementia associated with haemochromatosis: a report of two cases. Eur J Neurol 1997;4:318-22.

6 Berg D, Hoggenmuller U, Hofmann E, et al. The basal ganglia in haemochromatosis. Neuroradiology 2000;42:9-13.

7 Krieger J, Schroeder C. Iron, brain and restless legs syndrome. Sleep Med Rev 2001; 5:277-86.

8 Allen RP, Barker PB, Wehrl F, et al. MRI measurement of brain iron in patients with restless legs syndrome. Neurology 2001;56:263-5.

9 Earley CJ, Connor JR, Beard JL, et al. Abnormalities in CSF concentrations of ferritin and transferrin in restless legs syndrome. Neurology 2000;54:1698-700

10 Connor JR, Boyer PJ, Menzies SL, et al. Neuropathological examination suggests impaired brain iron acquisition in restless legs syndrome. Neurology 2003;61:304-9.

11 Graham JM, Paley MN, Grunewald RA, et al. Brain iron deposition in Parkinson's disease imaged using the PRIME magnetic resonance sequence. Brain 2000;123:2423-31. 